\title{
Meteor Detection using Deep Convolutional Neural Networks
}

\author{
Thiago C. L. Marsola* Ana C. Lorena** \\ *Itaú Unibanco, SP, (e-mail: thiago.marsola@itau-unibanco.com.br). \\ ** Department of Computer Engineering, Technological Institute of \\ Aeronautics, SP (e-mail: aclorena@ita.br)
}

\begin{abstract}
This paper describes an application of a pre-trained Deep Convolutional Neural Network to detect meteors from night sky images. The dataset is relatively small, composed of labeled images of meteors and non-meteors from the night sky. Techniques like data augmentation were used to create data artificially, and a dropout layer was introduced to prevent overfitting at the training augmented dataset. The performance obtained by the methods using traditional five-fold cross-validation along with changing of the images for black and white, compared with the ones obtained using only the training and validation partitions and RGB colors, achieved a higher mean accuracy of $84.35 \%$.

Resumo: Este artigo descreve a aplicação de uma rede neural convolucional profunda prétreinada para detectar meteoros a partir de imagens do céu noturno. O conjunto de dados é relativamente pequeno, composto de imagens rotuladas de meteoros e não-meteoros. Técnicas como aumento de dados foram usadas para criar dados artificialmente, e uma camada de dropout foi introduzida para evitar overfitting no dataset aumentado para treinamento. $\mathrm{O}$ desempenho obtido pelos métodos usando a validação cruzada com cinco partições, juntamente com a mudança das imagens para preto e branco, em comparação com os obtidos usando apenas as partições de treinamento e validação e cores RGB, alcançou uma precisão média maior de $84,35 \%$
\end{abstract}

Keywords: Artificial Neural Networks; Meteor Detection; Patter Recognition; Transfer Learning.

Palavras-chaves: Redes Neurais Artificiais; Detecção de Meteoros; Reconhecimento de Padrões; Aprendizado por Transferência.

\section{INTRODUCTION}

Meteor detection is an important area to explore for many different reasons. The level of information contained in a meteor can provide relevant data about stellar evolution since meteorites contain grains of dust ("stardust") that may be produced by stars before the formation of our Solar System. Also, by analyzing the concentrations of multiple elements and their isotopes in meteorites, the chemical composition of the Solar System can be inferred and the order in which different components in meteorites, their parent bodies, and the Solar System are formed Chodas (2015). These are some reasons of why aerospace agencies, such as NASA, finances centers for detection of meteors, like the Center for Near-Earth Objects Studies (CNEOS) in the California Institute of Technology Silva et al. (2018).

With the relevance of meteor detection for scientific research explained, Brazil possesses some citizen science initiatives also dedicated to the monitoring of meteors, such as EXOSS (Exploring the Southern Sky) ${ }^{1}$ and BRAMON (Brazilian Meteor Observation Network) ${ }^{2}$. EXOSS has

\footnotetext{
* Reconhecimento do suporte financeiro deve vir nesta nota de rodapé.

1 http://exoss.org

2 http://sonotaco.com/e-index.html
}

more than 50 stations equipped with low cost CCTV (Closed-Circuit Television) cameras that constantly monitor the sky at night, automatically capturing possible images of meteors and meteorites and non-meteors Silva et al. (2018). Combining data from multiple stations and using a specific software, the EXOSS project is capable of acquiring information about the trajectory, velocity, and the most probable impact point of the meteor. The cameras inevitably capture other events such as the passage of animals and insects, airplanes, fireworks, and atmospheric events. Those captures must be classified and filtered, a task which is usually performed manually. The interest is to accurately identify those images which contain meteor records. The usual procedure adopted by the specialists for that is to inspect the images periodically in order to discard the non-meteors Galindo and Lorena (2018).

For this work, part of the dataset used was provided by researchers from UNIVAP (Universidade do Vale do Paraíba), and another part were obtained through images from another EXOSS stations, resulting in 310 images of meteors and 185 images of non-meteors. Two examples of capturing images can be viewed in Fig.1 and 2 .

Deep Convolutional Neural Networks (CNN) are capable of achieving record-breaking results on highly challenging 


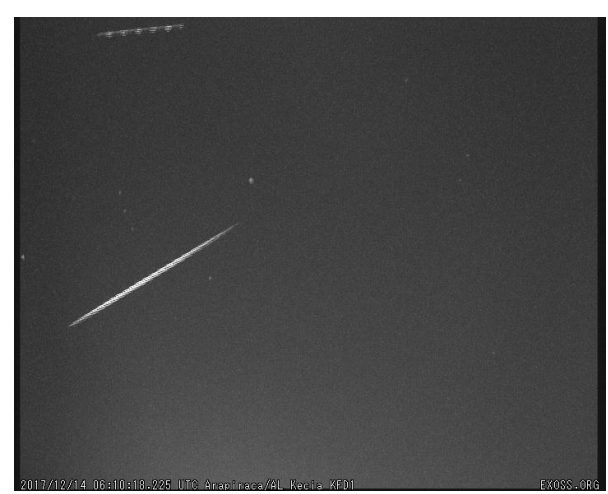

Figura 1. Example of a meteor.

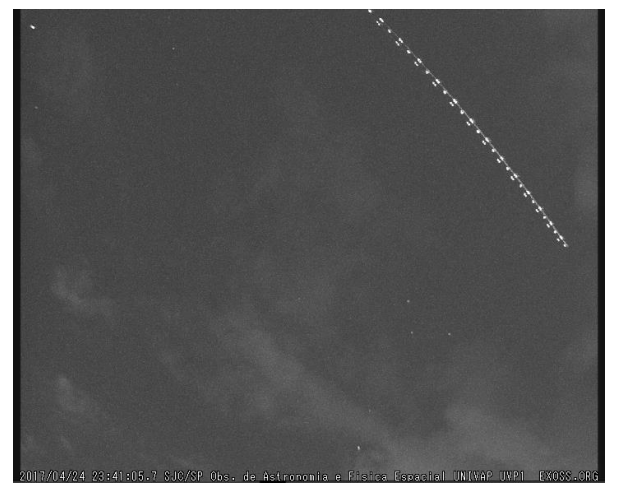

Figura 2. Example of a non-meteor.

datasets Krizhevsky et al. (2012). Also, they are capable of dealing with raw image data without any feature engineering, surpassing human performance in image classification in some datasets $\mathrm{He}$ et al. (2015). With large datasets combined with highly modern GPUs, these deep networks provide state-of-art results, with a high predictive accuracy. However, if there is a limited access of large quantities of data, this could result in a overfitting, resulting in low predictive accuracy for the test data.

Thereby, this paper presents a set of experiments performed using deep CNN's to the problem of detecting the presence of meteors in captures night sky images. Since the considered dataset is small, one strategy to surpass this problem was the use of transfer learning. Using the learned features of a trained deep network on a large dataset, with a five-fold cross validation, it is possible to fine tune the network for the given relatively small meteor dataset, achieving $84.35 \%$ of accuracy in the detection of meteors.

The remainder of this paper is organized as follows: Section II presents the related work. The experimental settings and tools used for the purpose of this paper is described in Section III. The results obtained by the convnet are presented in Section IV. Lastly, Section V presents the conclusions and some future works.

\section{RELATED WORK}

Several approaches have been applied to the problem of image classification using deep neural networks. One is the train of a network on ImageNet (where classes are mostly animals and everyday objects) and then re-use this trained network for something as remote as identifying furniture items in images Chollet (2017). Also, different sets of networks have been applied using the transfer learning features from ImageNet Rastegari et al. (2016). It is important to have in mind the generality versus specificity of neurons in each layer of a deep CNN's, since transferability is negatively affected by two distinct issues: the specialization of higher layer neurons to their original task and the optimization difficulties related to splitting networks between co-adapted neurons Yosinski et al. (2014). However, an increased network depth may not harm predictive performance results, optimizing some factors such as its architecture, distribution of the training data and parameters of feature extraction such as layer of the trained ConvNet Azizpour et al. (2016).

The effect of increasing depth using an architecture with very small convolution filters was also proved significant to the prior-art configurations, incrasing the depth to $16-$ 19 weight layers Simonyan and A. (2014). Among the use of other classifiers working with CNNs, the Support Vector Machine (SVM) achieved great prediction results when using the features obtained by a CNN trained with the ImageNet dataset, outperforming other specific architectures in tasks such as object classification and attribute detection Razavian et al. (2014). Moreover, there are experiments that the learned features are compared to point detectors and descriptors that achieved success in object recognition Donahue et al. (2014).

In addition, in the area of medical image classification where CNNs are widely used due to their high accuracy, there are currently three major training techniques: training the CNN from scratch, using off-the-shelf pre-trained CNN features, and conducting unsupervised CNN pretraining with supervised fine-tuning. One effective method that has been used recently is transfer learning, i.e., finetuning CNN models (supervised) pre-trained from one given image dataset to other image tasks. These type of models contain 5 thousand to 160 million parameters, with a variation of the number of layers. With this, the transfer learning from pre-trained ImageNet (via fine-tuning) was tested and verified Hoo-Chang et al. (2016).

Finally, in the area of classifying meteor images, in Zoghbi et al. (2017) it is described an effort to automate the CAMS (Cameras for Allsky Meteor Surveillance) data reduction pipeline to discriminate meteors from other types of objects detected. Basically, the method supports the processing of the data collected every night from lowlight video observations, and makes results available to the observer the following day. Those detections are classified as meteors or non-meteors using two methods: a CNN, and a long short term memory network (LSTM).

\section{METHODOLOGY}

In order to provide a better comprehension of the proposed classification strategy, a brief theoretical revision is stated. Regular CNN's are formed by neurons with grouping weights in kernels, also known as filters or feature detectors. A kernel is basically a small matrix comprised of weights to be applied at an image comprised of pixels. Each filter is applied to the whole image in a process called convolution, resulting in an another image in which each pixel value is the result of a combination of pixel values 
from the original image resulted by the convolution, weighted by the kernel. Convolutions operate over 3D tensors (feature maps), with two spatial axes (height and width) as well as a depth axis (also called the channels axis). For an RGB image, the dimension of the depth axis is three, because the image has three color channels: red, green, and blue. For a black-and-white picture, like the MNIST digits, the depth is one (levels of gray) Chollet (2017).

Therefore, the results of this filters are multiplied by an activation function (ReLu, Hiperbolic Tangent, Sigmoid, and so on), resulting in another image called an activation map, indicating how much of the feature is detected by the filter in that point. Each map is interpreted as an image channel by the next kernels. The first filters learn general purpose features, such as the presence of shades, contours, edges and curves, while deeper filters will learn more abstract features such as bright, colors and abstract forms Chollet (2017). The use of a pooling layer reduces the size of activation maps between the other layers, and small activation maps of the last layers can be used as high level features for classical classification algorithms such as SVM.

In this work, a 6 layer neural network was used. The transfer learning technique was used considering the VGG16 neural network trained with the ImageNet dataset, since it is a common and widely used architecture that can achieve high accuracy in classification. For instance, in Szegedy et al. (2015) the classification and detection results using a deep CNN with ImageNet was $7.89 \%$ of error rate on ImageNet, surpassing human performance.

\section{EXPERIMENTAL SETTINGS}

The dataset used in all experiments is unbalanced, with a ratio of meteor to non-meteor images of approximately $2: 1$, and small compared with the one used in Galindo and Lorena (2018), so in order to verify overfitting, a repeated five-fold cross-validation was performed.

Also, before training, the convolutional base from the VGG16 network was freezed. The idea behind this strategy was freezing a layer or set of layers in order to prevent the update of weights during training. It is important to do this because if not the representations that were previously learned by the convolutional base will be modified during training. Since the dense layers on top are randomly initialized, very large weight updates would be propagated through the network, effectively destroying the representations previously learned.

In relation to the optimization algorithm, this work used the RMSProp optimizerRuder (2016). This algorithm has the purpose to avoid information from the past calculated gradients, introducing a decay rate into these past terms. The decay rate gives an idea of forgetting old steps, disregarding old gradients in order to take longer steps at a time. Usually, a weighted average is used between the new and old terms, and according to Chollet (2017), learning rate values with order of $10^{-5}$ provides good results. Thus, this work used RMSProp with a learning rate of $1 \times 10^{-5}$.

\subsection{Meteor Dataset}

The meteor dataset was composed by images captured by a monitoring station located at the Observatory of Astronomy and Space Physics from the University of Vale do Paraíba (UNIVAP), in São José dos Campos, SP, Brazil. They were recorded during the months of April and March, 2017 Galindo and Lorena (2018). A total of 122 images (41 from meteors and 81 from non-meteors) were captured and labeled by specialists. In order to increase this dataset, 220 labeled images of meteors and nonmeteors were obtained through the EXOSS website. This dataset of 400 images has also challenging characteristics, since it has a relative small quantity of images and contains noisy data (poor quality, street lights and non-relatable figures).

The target size of the each image contained in the trained dataset was resized to a vector of $224 \times 224$, and the train and test data generator rescaled the images by the factor $1 / 255$. The batch size used was equal to 20 images. In order to obtain more images artificially, the data augmentation technique was used. With this, each image from the entire dataset was zoomed in $20 \%$, rotated in 40 degrees, had a shift height and width range of $20 \%$, a shear range of 0.1 , and the filling mode is based on the nearest function, which means that each blank space from the shifted augmented image is resulted by the application of the latter techniques, that will be completed by the nearest pixels from the image itself.

\subsection{Pre-training}

The trained weights learned with ImageNet, combined with a 6 layer network for the classification task, imported from Keras. It was used the VGG16 model with 14.714.688 parameters, trained with the ImageNet dataset, and then, a flatten layer is introduced to make a one-dimensional output. With this, three dense layers are applied, each one with 32,16 , and 8 neurons. In order to simplify the images to diminish the effort of classification, the entire dataset was changed to black and white, dealing with only one channel instead of the three channel RGB immages. The resulting figures can be seen in Figures 3 and 4. Last, a dropout layer is applied to shut-down some neurons in order to increase the generalization capacity of the neural network, preventing overfitting. Another interpretation for the dropout layer is the training of a exponential quantity of neural networks that share more weights, combined into an efficient manner in a complete neural network with weights of smaller magnitude Chollet (2017).

The Global MaxPooling 2D layer is used to aggressively reduce the feature maps, analogous to strided convolutions. Max pooling consists of extracting windows from the input feature maps and outputting the max value of each channel. It works conceptually similar to convolution, except that instead of transforming local patches through learned linear transformation (the convolution kernel), it transforms those patches with max tensor operation. A significant difference from convolution is that max pooling is usually done with $2 \times 2$ windows and stride 2 , in order to downsample the feature maps by a factor of 2 . Lastly, it is important to notice that the max pooling technique is 
1.0

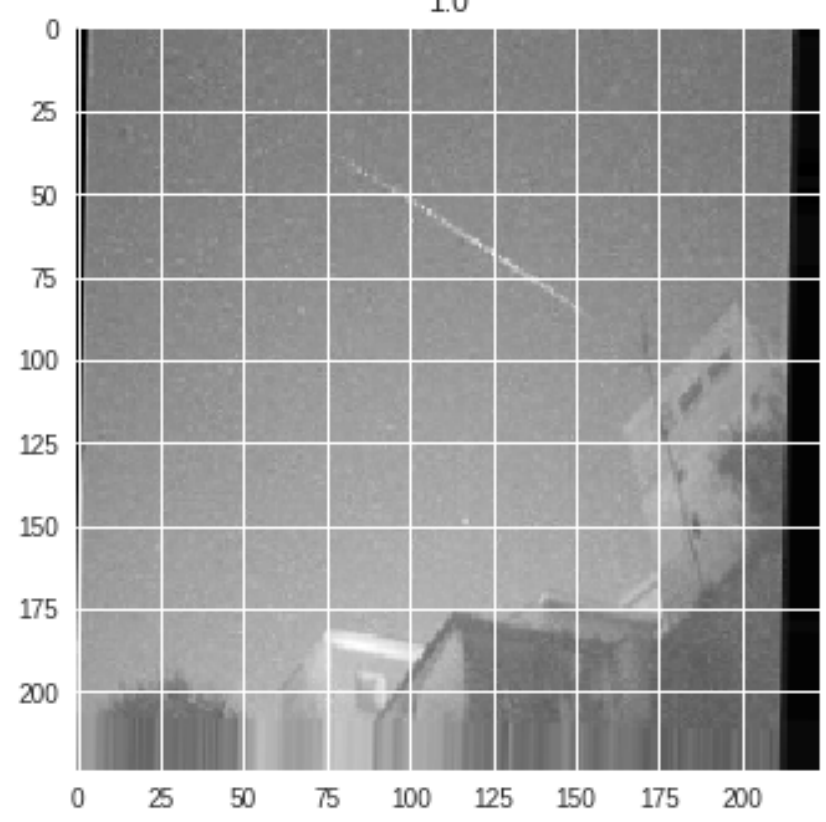

Figura 3. Black and white figure of a meteor.

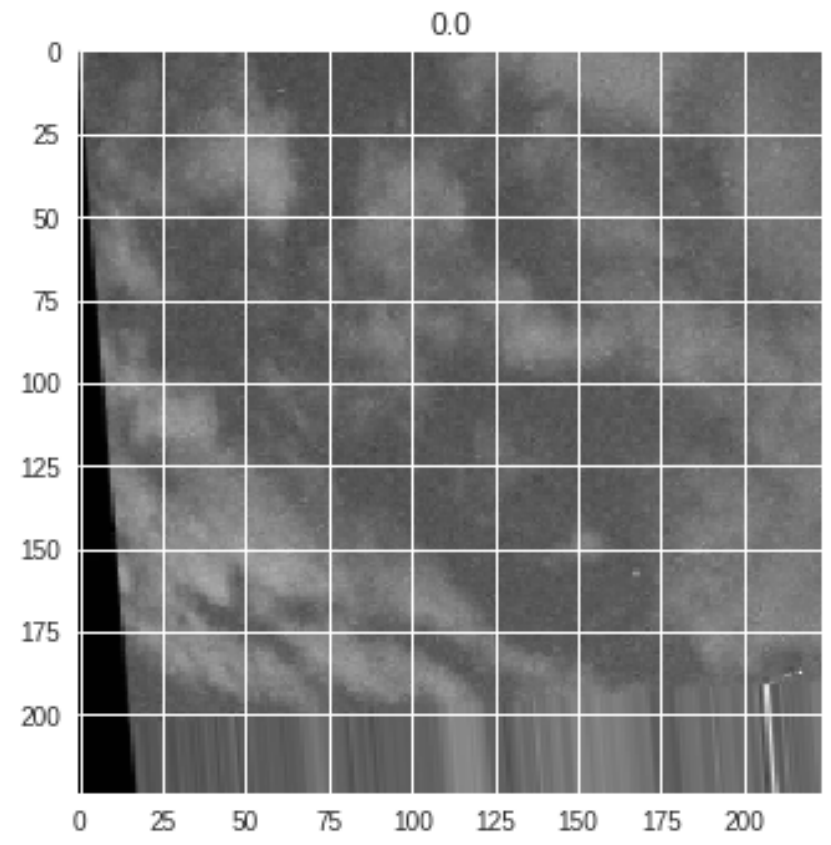

Figura 4. Black and white figure of a non-meteor.

not the only way to achieve such downsampling. Another alternative is the use of strides in the prior convolution layer using average pooling instead, where each local input patch is transformed by taking the average value of each channel over the patch, rather than the max. Nevertheless, the max pooling tends to work better than those alternative solutions Chollet (2017).

The dropout regularization capacity of generalization is greater than other techniques of regulation already proposed in the literature, such as: (1) stopping the training early in minimizing the error along with a validation set; (2) penalize the magnification of the vector of weights via the L1 standard (LASSO) or standard L2 (ridge regression); (3) promote weight sharing. A batch size of twenty images was used, and at each training time or batch, a lean neural network is randomly sampled from the complete neural network. Thus, training a neural network applied with $n$ dropout units can be interpreted as $2^{n}$ training neural networks that share synaptic weights Srivastava et al. (2014). The output is composed of a dense layer with only one neuron. The whole model can be viewed in Fig. 5.

\begin{tabular}{|c|c|c|}
\hline Layer (type) & Output Shape & Param \# \\
\hline $\begin{array}{l}================== \\
\text { vgg16 (Model) }\end{array}$ & $\begin{array}{l}================= \\
\text { (None, } 7,7,512)\end{array}$ & $\begin{array}{l}========= \\
14714688\end{array}$ \\
\hline flatten_3 (Flatten) & (None, 25088) & $\theta$ \\
\hline dense_5 (Dense) & (None, 32) & 802848 \\
\hline dense_6 (Dense) & (None, 16) & 528 \\
\hline dense_7 (Dense) & (None, 8) & 136 \\
\hline dropout_1 (Dropout) & (None, 8) & $\theta$ \\
\hline 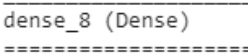 & 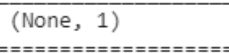 & 9 \\
\hline $\begin{array}{l}\text { Total params: } 15,518 \\
\text { Trainable params: } 86 \\
\text { Non-trainable params }\end{array}$ & & \\
\hline
\end{tabular}

Figura 5. Model layers.

\subsection{Evaluation}

The evaluation metrics used in this work were the log-loss and the accuracy.

The first one evaluates the accuracy of a classifier by penalizing false classifications. Thus, minimizing the logloss is similar to maximizing the classifier's accuracy. As a result, a perfect classifier has a log-loss of zero, while the remaining classifiers have progressively larger values. Logloss is based on uncertainty, so the classifier predictions must be probabilities Lorena et al. (2018). For a binary classification problem, the log-loss expression is:

$$
-\frac{1}{N} \sum_{i=1}^{N}\left[y_{i} \log \left(p_{i}\right)+\left(1-y_{i}\right) \log \left(1-p_{i}\right)\right]
$$

where $N$ is the data set size, $y_{i}$ is the correct class of instance $i, p_{i}$ is the predicted probability for instance $i$, and $\log$ is the natural logarithm. As the accuracy, which is the second metric used, is calculated by the ratio of number of correct predictions to the total number of input samples.

\subsection{Fine-Tunning}

The fine tuning procedure was implemented to improve significantly the performance of the CNN, as seen in Table 1. This technique is based for model reuse, complementary to feature extraction, consisting in unfreezing a few of the top layers of a frozen model base used for feature extraction, and jointly training both the newly added part of the model (in our case, the fully-connected classifier) and these top layers. This is called "fine-tuning"because it slightly adjusts the more abstract representations of the model being reused, in order to make them more relevant for the problem at hand Chollet (2017). 
Tabela 1. Accuracy using or not fine-tunning procedure.

\begin{tabular}{|c|c|}
\hline & Accuracy (acc) \\
\hline No fine-tunning & $79.89 \%$ \\
With fine-tunning & $84.35 \%$ \\
\hline
\end{tabular}

\section{RESULTS}

Different types of models were experimented, varying hyperparameters like the size of the batches, the activation functions, the optimization algorithm, and the number of dense layers. Therefore, the best model resulted in a $84.35 \%$ accuracy taking into acount the average of the fivefold cross-validation, with batch size of 20 images, three dense layers of 32,16 and 8 neurons, with the first and third ones composed by the hyperbolic tangent function, and the second one composed by the relu function, along with the RMSProp algorithm.

In addition, the early stopping technique was implemented as a callback function. Callbacks are functions that can be applied at certain stages of the training process, such as at the end of each epoch. The patience argument define that we wanted to monitor the validation loss at each epoch, and if after this loss has not improved after 3 epochs, the training is interrupted.

These parameters with the corresponding accuracy indicate that the sets of features that were used to represent the examples of the proposed dataset were sufficiently informative to distinguish most of the images containing meteors from the ones that do not contain meteors.

\section{CONCLUSIONS}

This work presented one of the most used techniques in the area of image classification problems, the deep convolutional neural networks, applied to classify images containing meteors. With a small dataset, the technique of data augmentation was used to create data artificially. Also, the fine tuning technique was used and improved the accuracy on the test dataset in almost $5 \%$. All experiments were performed on the cloud GPU platform Google Colab.

Also, the results obtained using a five-fold cross-validation were different from the ones obtained using the original training and testing partitions of the dataset. This result could be explained by the differences from each batch of images, since the time, period of the year, and the city that the picture from the night sky was taken is very different from each other. The transformation for the black and white images using the "grayscale"color mode allowed the CNN to extract and classify a smaller quantity of features, since it has now only one channel instead of the RGB three channel.

The investigation presented was in accordance with the literature, since pre-training a deep network with images containing black and white pixels it was possible to achieved a good accuracy of $84.35 \%$ using fine-tunning on this dataset. In Galindo and Lorena (2018), an analogous strategy of classification was performed using transfer learning, with a dataset with more than 1000 images of meteors and non-meteors, achieving an accuracy of more than $93 \%$.
For future works, an interesting idea is to use other types of CNN's, pre-trained with other datasets, like ResNet, Xception, Inception and so on, to capture the differences of each deep neural network in working with this type of dataset.

\section{ACKNOWLEDGMENT}

This work was supported by Itaú Unibanco.

\section{REFERENNCIAS}

Azizpour, H., Razavian, S.H., Sullivan, J., Maki, A., and Carlsson, S. (2016). Factors of transferability for a generic convnet representation. IEEE transactions on pattern analysis and machine intelligence, 38(9), 17901802.

Chodas, P. (2015). Overview of the jpl center for neo studies (cneos). AAS/Division for Planetary Sciences Meeting Abstracts, 47.

Chollet, F. (2017). Deep learning with python.

Donahue, J., Jia, Y., Vinyals, O., Hoffman, J., Zhang, N., Tzeng, E., and Darrel, T. (2014). Decaf: A deep convolutional activation feature for generic visual recognition. In In International conference on machine learning, 647-655.

Galindo, Y. and Lorena, A.C. (2018). Deep Transfer Learning for Meteor Detections. In ENIAC - Encontro Nacional de Inteligência Artificial e Computacional.

He, K., Zhang, X., Ren, S., and Sun, J. (2015). Delving deep into rectifiers: Surpassing human-level performance on imagenet classification. In Proceedings of the IEEE international conference on computer vision, 1026-1034.

Hoo-Chang, S., Roth, H.S., Gao, M., Lu, L., Xu, Z., Nogues, I., and Summers, R.M. (2016). Deep convolutional neural networks for computer-aided detection: CNN architectures, dataset characteristics and transfer learning. IEEE transactions on medical imaging, 35(5).

Krizhevsky, A., Sutskever, I., and Hinton, G.E. (2012). Imagenet classification with deep convolutional neural networks. Advances in neural information processing systems, 1097-1105.

Lorena, A.C., Kaster, D.S., Cerri, R., Faria, E.R., and de Melo, V.V. (2018). Can I make a wish?: a competition on detecting meteors in images. In Symposium on Knowledge Discovery, Mining and Learning - KDMILE. Rastegari, M., Ordonez, V., Redmon, J., and Farhadi, A. (2016). Imagenet classification using binary convolutional neural networks. In European Conference on Computer Vision, 525-542.

Razavian, S.A., Azizpour, H., Sullivan, J., and Carlsson, S. (2014). Cnn features off-the-shelf: an astounding baseline for recognition. In Proceedings of the IEEE conference on computer vision and pattern recognition workshops, 806-813.

Ruder, S. (2016). An overview of gradient descent optimization algorithms.

Silva, R.M., Lorena, A.C., and Almeida, T.A. (2018). Detecting the presence of meteors in images: new collection and results. In BRACIS - Brazilian Conference on Intelligent Systems.

Simonyan, K. and A., Z. (2014). Very deep convolutional networks for large-scale image recognition. 
Srivastava, N., Hinton, G.E., Krizhevsky, A., Sutskever, I., and Salakhutdinov, R. (2014). Dropout: a simple way to prevent neural networks from overfitting. The Journal of Machine Learning Research, 15(1), 1929-1958.

Szegedy, C., Liu, W., Jia, Y., Sermanet, P., Reed, S., Anguelov, D., and Rabinovich, A. (2015). Going deeper with convolutions. In Proceedings of the IEEE conference on computer vision and pattern recognition, 1-9.

Yosinski, J., Clune, J., Bengio, Y., and Lipson, H. (2014). How transferable are features in deep neural networks? Advances in neural information processing systems, $3320-3328$

Zoghbi, S., De Cicco, M., Ordonez, A.J., Stapper, A.P., Collison, S.P., Gural, S.P., Ganju, S., Galache, J.S., and Jenniskens, P. (2017). Searching for Long-Period Comets with Deep Learning Tools. In Workshop on Deep Learning for Physical Sciences. 